\title{
ANTIBIOTIC SUSCEPTIBILITY TESTING FOR ESCHERICHIA COLI CAUSING URINARY TRACT INFECTIONS IN SOKOTO METROPOLIS
}

\author{
MUHAMMAD NURA UMAR ${ }^{1,2 *}$, BATURE MUSTAPHA ${ }^{3}$, NUHU TANKO ${ }^{2}$, NAFIU AMINU $^{1,2}$ \\ ${ }^{1}$ Department of Pharmaceutical Technology, School of Pharmaceutical Sciences, Universiti Sains Malaysia, Penang, Malaysia. ${ }^{2}$ Department \\ of Pharmaceutics and Pharmaceutical Microbiology, Faculty of Pharmaceutical Sciences, Usmanu Danfodiyo University, Sokoto, Nigeria. \\ ${ }^{3}$ Department of Surgery, Faculty of Clinical Sciences, Usmanu Danfodiyo University Sokoto, Nigeria. Email : chemistnuratbw@gmail.com
}

Received: 02 July 2017, Revised and Accepted: 03 March 2018

ABSTRACT

Objective: The study was designed to diffuse awareness on the prevalence of Escherichia coli as a causative agent of urinary tract infection (UTI) in Sokoto metropolis as well as to determine the susceptibility to commonly used antibiotics in Specialist Hospital Sokoto (SHS). This is also to raise awareness of the risk of giving antibiotics and their direct impact on the outcome analysis of UTIs.

Methods: This study was conducted at SHS, and ethical approval to carry out the study was obtained from the Ethical Committee of the hospital. Informed consent was obtained from each participant. Early morning, mid-stream clean catch urine samples were collected by patients in sterile disposable containers. The antibiotic susceptibility of the isolates was determined against 10 commonly prescribed antibiotics in SHS using the modified Kirby-Bauer disc agar diffusion.

Results: A total of 86 urine samples were analyzed over 2 months, and 34 were culture positive giving an isolation rate of $39.5 \%$, while 48 were culture negative giving a rate of $55.8 \%$, and 4 (4.7\%) were undecided. A total of 16 isolates were $E$. coli (47.1\%), while 18 accounts for others (52.9\%). The results of antimicrobial susceptibility profile to 10 antibiotics showed that $E$. coli displayed high susceptibility to vancomycin ( $91.6 \%)$, followed by amikacin $(89.2 \%)$ and then meropenem $(88.0 \%)$, while high rate of resistance was found in nalidixic acid $(81.2 \%)$, followed by co-trimoxazole (73.3\%) and then norfloxacin (76.2\%).

Conclusion: When there is an adequate detection of E. coli and other uropathogens, it will aid in selecting the appropriate antimicrobial therapy and this will also serve as a means of infection control. This will go a long way in reducing the cost of treatment and threat of resistance as witnessed in the management of some uropathogens.

Keywords: Antibiotic susceptibility, Escherichia coli, Uri nary tract infections.

(c) 2018 The Authors. Published by Innovare Academic Sciences Pvt Ltd. This is an open access article under the CC BY license (http://creativecommons. org/licenses/by/4. 0/) DOI: http://dx.doi.org/10.22159/ajpcr.2018.v11i6.21086

\section{INTRODUCTION}

Urinary tract infections (UTIs) have become the most common hospitalacquired infection, accounting for as many as 35\% of nosocomial infections, and it is the second most common cause of bacteremia in hospitalized patients $[15,18,20,21]$. These infections are found commonly in women than in men. The incidence in women within the age of $20-40$ years ranges from $25 \%$ to $30 \%$ and up to $4-43 \%$ in elderly women above 60 years of age [3].

It is one of the most common bacterial diseases worldwide $[5,8,16,28]$ that is characterized by a wide range of symptoms from mild irritative voiding to bacteremia, sepsis, or even death $[16,26]$.

Bacteria are the major causative organisms and are responsible for more than $95 \%$ of UTI cases $[16,25]$. About $80-85 \%$ of UTIs are caused by Gram-negative bacteria $[12,29]$. UTIs have different names, depending on which the part of the urinary tract is infected. UTIs are classified as uncomplicated or complicated. It has been observed that despite the widespread availability of antibiotics, UTI remains the most common bacterial infection in the human population $[9,22]$.

It has been reported that UTI can occur in any part of the urinary tract and is caused by the retrograde ascent of bacteria from the fecal flora through the urethra to the bladder and kidney [22]. This is most, especially, in the females who have a shorter and wider urethra and is more transversed by microorganisms $[13,17]$. In most cases, bacteria travel to the urethra and multiply causing kidney infection if not treated [4].

However, there are some urinary tract diseases that are not associated with urinary infection but often treated with antibiotics, a practice that leads to antibacterial resistance due to improper diagnosis of UTIs. The most commonly reported are interstitial cystitis (IC) and overactive bladder (AOB). IC has been described [25] as the complaint of suprapubic pain related to bladder filling, accompanied by other symptoms such as increased day and night time frequency, in the absence of proven urinary infection or other obvious pathology. The prevalence of IC in the developed countries is 197 and 41 in every 100,000 women and men respectively. AOB has also been described [25] as the symptom complex of urinary urgency, usually accompanied by frequency and nocturia, with or without urgency urinary incontinence, in the absence of urinary tract infection or other obvious pathology. The prevalence of $\mathrm{OAB}$ in men and women is $10.8 \%$ and $12.8 \%$ in a population of 100,000 respectively.

A research by Goldman and Huskins [10] suggested that the improper and uncontrolled use of many antibiotics resulted in the occurrence of antimicrobial resistance, which became a major health problem worldwide. Another author, Manikandan et al. [19], also reported that the "widespread use and more often the misuse of antimicrobial drugs have led to a general rise in the emergence of resistant bacteria." Therefore, the diagnosis of UTI is usually made based on the presence of signs and symptoms and confirmed by culture examination with significant bacteriuria supported by high-level pyuria $[16,22]$. 
Escherichia coli is the most common cause of UTI among virtually every patient group and accounts for $80-90 \%$ of cases of uncomplicated pyelonephritis and cystitis [27].

The current study aimed to determine and disseminate awareness on the prevalence of $E$. coli associated with UTI as well as their susceptibility pattern in Sokoto metropolis.

\section{METHODS}

Approval to carry out the study was obtained from the Ethical Committee of Specialist Hospital Sokoto (SHS), and informed consent was obtained from each participant (Appendix I)

\section{Media preparation}

The media used in this work include cysteine lactose electrolyte deficient (CLED) agar, and nutrient agar (NA), and Mueller-Hinton Agar (MHA), all sourced from Hi Media, India. The media were prepared based on manufacturer's instruction and sterilized by autoclaving for $15 \mathrm{~min}$ at $121^{\circ} \mathrm{C}$.

\section{Sample collection}

Our study included patients (male and female) of all ages who attended the outpatient department with evidence or symptoms of UTI as determined by the physician. Early morning, mid-stream clean catch urine samples were collected by patients in sterile disposable containers with screw caps. Before urine collection, patients were counseled on how to collect the urine sample by observing all aseptic conditions to avoid contamination.

\section{Isolation and culturing of urine samples}

Sterile Petri dishes containing $20 \mathrm{~mL}$ prepared CLED agar were allowed to set and their surfaces dried in an incubator at $37^{\circ} \mathrm{C}$ for $5 \mathrm{~min}$. Urine samples were inoculated on CLED agar using calibrated wire loop and allowed to stay for $30 \mathrm{~min}$ and incubated in aerobic condition for 18$24 \mathrm{~h}$ at $37^{\circ} \mathrm{C}$. Plates without any colony at the end of $18-24 \mathrm{~h}$ incubation were discarded. Samples with counts up to and $>10^{5} \mathrm{CFU} / \mathrm{mL}$ were counted microscopically and considered positive for further analysis.

\section{Characterization of isolates}

Isolates were purified by single colony isolation unto NA plates and incubated at $37^{\circ} \mathrm{C}$ for $18-24 \mathrm{~h}$. Isolates from pure culture were characterized by Gram-staining followed by different biochemical tests (indole production test, motility test, and triple sugar iron agar test) were performed to confirm the $E$. coli causing UTI.

\section{Antibiotic susceptibility testing}

The antibiotic susceptibility of the isolates was determined against 10 commonly prescribed antibiotics in SHS using the modified Kirby-Bauer disc agar diffusion [6,7]. The discs (Oxoid, UK) were meropenem (MER, $10 \mu \mathrm{g}$ ), amikacin (AMK, $30 \mu \mathrm{g}$ ), vancomycin (VA, $10 \mu \mathrm{g}$ ), amoxicillin/ clavulanic acid (AMC, $30 \mu \mathrm{g}$ ), ciprofloxacin (CIP, $5 \mu \mathrm{g}$ ), norfloxacin (NOR, $10 \mu \mathrm{g}$ ), cotrimoxazole (SXT, $25 \mu \mathrm{g}$ ), nitrofurantoin (F, $300 \mu \mathrm{g}$ ), gentamicin (CN, $30 \mu \mathrm{g})$, and nalidixic acid (NA, $30 \mu \mathrm{g})$.

A fresh subculture of isolates was prepared on MHA and incubated at $37^{\circ} \mathrm{C}$ for $18-24 \mathrm{~h}$. With the aid of a wire loop, 4-5 well-isolated colonies of similar appearance were picked and transferred into the tube of sterile normal saline. The inoculum was emulsified inside the tube to avoid clumping of the cells. The inoculums were adjusted to 0.5 McFarland (McFarland 0.5 equals approximately $10^{8} \mathrm{CFU} / \mathrm{mL}$ ).

Within 15 min of preparing the adjusted inoculums, a sterile cotton swab was dipped into the inoculums. The swab was rotated several times and pressed firmly on the inside of the tube above the fluid level to remove excess inoculums from the swab.

The swab was streaked over the entire surface of the MHA plate, rotating the plate approximately $60^{\circ} 3$ times to ensure confluent growth. Inoculation was completed by running the swab around the rim of the agar. Excess moisture on the agar surface was allowed to be absorbed before applying the antimicrobial discs.
The disc was placed $20 \mathrm{~mm}$ center to center on the surface of the agar using a sterile needle. The plates were allowed to stay for 20-30 min to allow for pre-diffusion. The plates were incubated at $37^{\circ} \mathrm{C}$ for $18-24 \mathrm{~h}$.

Following incubation, the diameter of the zones of growth inhibition was measured to the nearest millimeter using a ruler, including the diameter of the disc in the measurement. Results were interpreted using the CLSI Guidelines (2006)

\section{RESULTS AND DISCUSSION}

A total of 86 urine samples were analyzed over 2 months' period and 34 were culture positive giving an isolation rate of $39.5 \%$, while 48 were culture negative giving a rate of $55.8 \%$, and $4(4.7 \%)$ were neither decided, due to probably contamination as shown in Fig. 1 . A total of 16 isolates were E. coli (47.1\%), while 18 accounts for others (other Gramnegative, 11 and Gram-positive, 7) as shown in Fig. 2.

The percentage susceptibility of E. coli causing UTI is shown in Table 1 . The antimicrobial susceptibility profile results were interpreted according to the CLSI, 2006 interpretative chart.

Different bacterial pathogens were reported to cause UTI with many reporters concluded that E. coli and Klebsiella spp. were found to be predominant in causing the UTI among patients. Hence, the present study was conducted and focused only on E. coli to determine its sensitivity and antibiotic resistance against ten commonly prescribed

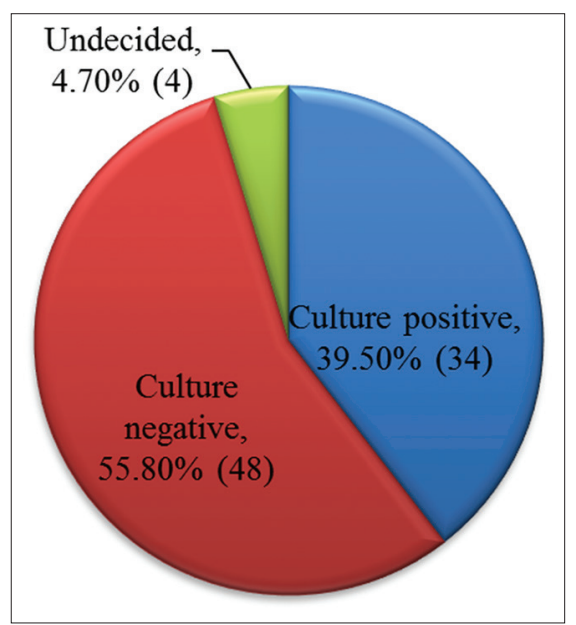

Fig. 1: Percentage distribution for the presence or absence of uropathogens from urine culture

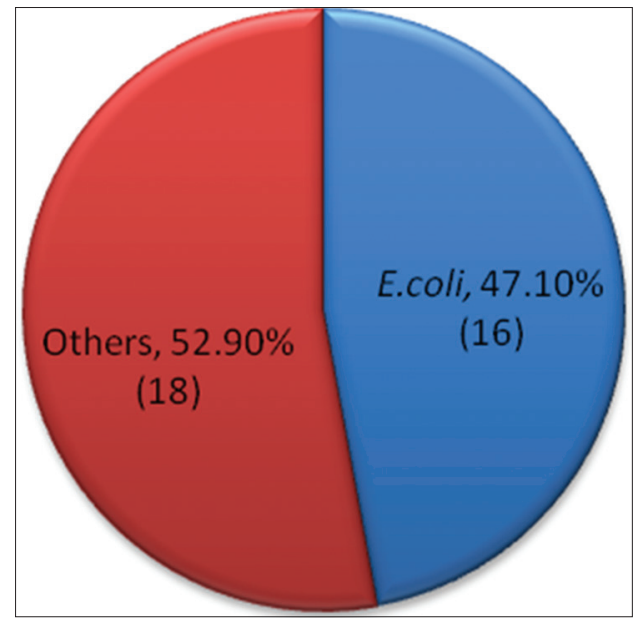

Fig. 2: Percentage distribution of uropathogens among culture positive 
Table 1: Percentage susceptibility of $E$. coli causing UTI

\begin{tabular}{lll}
\hline \multirow{2}{*}{ Antimicrobial agents } & \multicolumn{2}{l}{ Percentage susceptibility } \\
\cline { 2 - 3 } & Susceptible & Resistant \\
\hline Meropenem & 88.0 & 12.0 \\
Amikacin & 89.2 & 10.8 \\
Vancomycin & 91.6 & 8.4 \\
Amoxicillin/clavulanate & 81.4 & 18.6 \\
Norfloxacin & 23.2 & 76.8 \\
Co-trimoxazole & 21.8 & 78.2 \\
Nitrofurantoin & 71.4 & 28.6 \\
Gentamicin & 63.8 & 36.2 \\
Nalidixic acid & 18.8 & 81.2 \\
\hline
\end{tabular}

UTI: Urinary tract infection, E. coli: Escherichia coli

and commercially available antibiotics.

Muraleetharan and Viswanathan [20] reported that a total of 217 uropathogens were screened and found that E. coli (49.8\%) was the most common organism. This report strongly supports the present study as E. coli $(47.10 \%)$ was found to be the most prevalent Gramnegative bacteria among patients with evidence or symptoms of UTI as determined by the physician. This result is also consistent with reports from other studies [2,11,14,17,19,20,23,24]. Al-Jebouri and Mdish [1] highlighted reasons as to why $E$. coli is the most common cause of UTI may be due to certain virulence factors such as hemolysin production and presence of fimbriae.

E. coli from our study was generally resistant to nalidixic acid (81.2\%), cotrimoxazole (78.2\%), and norfloxacin $(76.2 \%)$. The high rate of resistance to nalidixic acid, cotrimoxazole, and norfloxacin is consistent with reports that these agents are the most commonly prescribed, cheaper, and easily available in the hospital and community pharmacies $[18,22,29]$. The results of the antimicrobial susceptibility profile to 10 commonly prescribed antibiotics showed that E. coli displayed a high susceptibility to vancomycin (91.6\%), followed by amikacin (89.2\%) and then meropenem (88.0\%).

This study was carried out to investigate and diffuse awareness on the prevalence of $E$. coli as a causative agent of UTI in Sokoto metropolis as well as to determine the susceptibility. This is to raise awareness of the risk of giving antibiotics and their direct impact on the outcome analysis of UTIs.

\section{CONCLUSION}

It is alarming that E. coli is the most common organism causing UTI among patients in SHS and found resistant to $30 \%$ of the commonly prescribed antibiotics. Therefore, treatment should be given only after culture and sensitivity test have been performed to prevent the misuse of antibiotics and reduce the risk of developing bacterial drug resistance. When there is an adequate detection of $E$. coli and other uropathogens, it will aid in selecting an appropriate antimicrobial therapy and this will also serve as a means of infection control.

\section{REFERENCES}

1. Al-Jebouri MM, Mdish SA. Antibiotic resistance pattern of bacteria isolated from patients with urinary tract infections in Iraq. Open J Urol 2013;3:124-31.

2. Amin M, Mehdinejad M, Pourdangchi Z. Study of bacteria isolated from urinary tract infection and determination of their susceptibility to antibiotics. Jundishapur J Microbiol 2011;2:118-23.

3. Basavaraj M, Jyothi P. Antimicrobial resistance of Pseudomonas aeruginosa Strains from patients with urinary tract infections in sbmpmc hospital Bijapur, India. Int J Pharm Pharm Sci 2014;6:479-81.

4. Bean DC, Daniel K, David WW. Antimicrobial resistance in community and nosocomial Escherichia coli urinary tract isolates, London 20052006. Ann Clin Microbiol Antimicrob 2008;24:7-13.

5. Chander A, Shrestha CD. Prevalence of extended spectrum betalactamase producing Escherichia coli and Klebsiella pneumoniae urinary isolates in a tertiary care hospital in Kathmandu, Nepal. BMC Res Notes 2013;6:487. Available from: http://www.biomedcentral. com/1756-0500/6/487.

6. Clinical Laboratory Standard Institute (CLSI). Perfomance Standards of Antimicrobial. Wayne, PA: Clinical and Laboratory Standards Institute; 2006.

7. Clinical and Laboratory Standards. Disc and dilution susceptibility tests for bacteria isolated from animal, approved standard. $3^{\text {rd }}$ ed., Vol. 28. Wayne, PA. Clinical and Laboratory Standards;

8. Cox CE. Nosocomial urinary tract infections. J Urol 1988;32:210-5

9. Durgesh DW, Tumane PM. Prevalence and antibacterial susceptibility pattern of Urinary tract infection causing human pathogenic bacteria. Asian J Biomed Pharm Sci 2012;2:1-3.

10. Goldman DA, Huskins WC. Control of nosocomial antimicrobialresistant bacteria: A strategy priority for hospitals worldwide. J Clin Infect Dis 1997;24:139-45.

11. Goswami R, Bal CS, Tejaswi S, Punjabi GV, Kochupillai N. Prevalence of urinary tract infection and renal scars in patients with diabetes mellitus. J Diabetes Res Clin Pract 2001;53:181-6.

12. Hussain N. Prevalence and susceptibility pattern of urinary pathogens. Biomedica 2012;18:76-9.

13. Inabo HI, Obanibi HB. Antimicrobial susceptibility of some urinary tract clinical isolates to commonly used antibiotics. Afr J Biotechnol 2006;5:487-9

14. Kehinde AO, Adedapo KS, Aimaikhu CO, Odukogbe AA, Olayemi O, Salako B. Significant bacteriuria among asymptomatic antenatal clinic attendees in Ibadan, Nigeria. J Trop Med Health 2011;39:73-6.

15. Khalifa AA, Alaa $\mathrm{OH}$, Abdalhamed MA. Urinary tract infection in sobrata, algmel cities in libya 2013. J Clin Microbiol 2014;3:205-2011.

16. Khoshbakt R, Salimi A, Shirzad AH, Keshavarz H. Antibiotic susceptibility of bacterial trains isolated from urinary tract infections in Karaj, Iran. Jundishapur J Microbiol 2013;6:86-90.

17. Kolawole AS, Kolawole OM, Kandaki-Olukemi YT, Babatunde SK, Durowade KA, Kolawole CF. Prevalence of urinary tract infections (UTI) among patients attending Dalhatu Araf Specialist Hospital, Lafia, Nasarawa State, Nigeria. Int J Med Med Sci 2009;1:163-7.

18. Lamido TZ, Ibrahim AR, Halima SM. Isolation and antibiotic sensitivity of Escherichia coli from pregnant and non-pregnant women attending the University of Maiduguri Teaching Hospital (UMTH), Maiduguri, Nigeria. Int J Biomed Health Sci 2010;6:315-22.

19. Manikandan S, Ganesapandian S, Manoj S, Kumaraguru AK. Antimicrobial susceptibility pattern of urinary tract infection causing human pathogenic bacteria. Asian J Med Sci 2011;3:56-60.

20. Muraleetharan M, Viswanathan T. Epidemiological studies on varying extended-spectrum $\beta$-lactamases producing uropathogenic bacteria. Int J Pharm Pharm Sci 2014;6:57-60.

21. Morgan MG, McKenzie H. Controversies in the laboratory diagnosis of community-acquired urinary tract infection. Eur J Clin Microbiol Infect Dis 1993;12:491-504.

22. Nuhu T. Retrospective studies on the prevalence of Uropathogens in Sokoto metropolis. Afr J Microbiol Res 2015;9:1366-70.

23. Okonko IO, Ijandipe LA, Ilusanya AO, Donbraye-Emmanuel OB, Ejembi J, Udeze AO, et al. Detection of urinary tract infection (UTI) among pregnant women in Oluyoro Catholic Hospital, Ibadan, SouthWestern Nigeria. Malays J Microbiol 2010;6:16-24.

24. Oladeinde BH, Omoregie R, Olley M, Anunibe JA. Urinary tract infection in a rural community of Nigeria. North Am J Med Sci 2011;3:75-7.

25. Ramesh N, Sumath CS, Kannan VR. Urinary tract infection and antimicrobial susceptibility pattern of extended-spectrum betalactamase producing clinical isolates. J Adv Biol Res 2008;2:78-82.

26. Ranjbar R, Haghi-Ashtiani M, Jafari NJ, Abedini M. The prevalence and antimicrobial susceptibility of bacterial uropathogens isolated from pediatric patients. Iran J Public Health 2009;38:435-41.

27. Safar F, Mohammed YA, Reza G, Behrooz N, Ailar N. Causative agents and antimicrobial susceptibilities of urinary tract infections in the North-West of Iran. Int J Infect Dis 2008;13:140-4.

28. Ullah F, Malik SA, Ahmed J. Antibiotic susceptibility pattern and ESBL prevalence in nosocomial Escherichia coli from urinary tract infections in Pakistan. Afr J Biotechnol 2009;8:3921-6. 
29. Venkatadri B, Arunagirinathan N, Rameshkumar MR, Sharmal KM, Agastian P. Multidrug resistant

Escherichia coli and Klebsiella pneumoniae from the urinary tract infections with special reference to extended spectrum -lactamase (ESBL) production. Int J Biol Pharm Res 2014;5:66-70. I 